\title{
The Feasibility of Flat, Portable and Wireless Device for Non-Invasive Peripheral Oxygenation Measurement over the Entire Body
}

\author{
Zehava Ovadia-Blechman ${ }^{*}$, Omri Gino', Liron Dandeker ${ }^{2}$, Nadav Sheffer ${ }^{1}$, \\ Erik Baltaxe ${ }^{3}$, Vered Aharonson 1,2,4 \\ ${ }^{1}$ Medical Engineering Department, Afeka Tel-Aviv Academic College of Engineering, Tel Aviv, Israel \\ ${ }^{2}$ Electrical Engineering Department, Afeka Tel-Aviv Academic College of Engineering, Tel Aviv, Israel \\ ${ }^{3}$ Pulmonary Diseases and Internal Medicine Institute, Sheba Medical Center, Tel Hashomer, Israel \\ ${ }^{4}$ School of Electrical and Information Engineering, University of the Witwatersrand, Johannesburg, South Africa \\ Email: "zehava@afeka.ac.il
}

Received 20 January 2016; accepted 21 March 2016; published 24 March 2016

Copyright (C) 2016 by authors and Scientific Research Publishing Inc.

This work is licensed under the Creative Commons Attribution International License (CC BY).

http://creativecommons.org/licenses/by/4.0/

(c) (i) Open Access

\section{Abstract}

Peripheral oxygenation level $\left(\mathrm{SpO}_{2}\right)$ can provide vital information on body functions. Continuous monitoring facilitates effective diagnosis and treatment and can even be lifesaving. Clinical device monitor $\mathrm{SpO}_{2}$ using a clip, and measure light transmission through the tissue. This method limits the body locations of the clip's placement and is sensitive to body movement, which hampers continuous $\mathrm{SpO}_{2}$ monitoring during wakefulness or sleep, thus decreasing its usability in clinics and its accessibility in homecare usage. We developed a portable, wireless, flat and low cost prototype for continuous monitoring of $\mathrm{SpO}_{2}$ that overcomes those limitations. The prototype enables convenient measurement in larger variety of body locations by spectrophotometric measurements of changes in the optical reflectance unlike other device that measure absorption through the tissue. The original design and signal processing enable reliable signal acquisition, synchronization and control. An Android's application was developed to provide a user friendly interface for results display on smartphones. The prototype's measurements were compared to commercial device that simultaneously measured heart rate frequency, transcutaneous oxygen tension $\left(\operatorname{tcPO}_{2}\right)$ and $\mathrm{SpO}_{2}$. The prototype's measurements accurately reflected changes caused by blood pulses, were correlated to the heart rate, and were sensitive to changes in oxygen saturation. Excellent real time behavior and synchronization were demonstrated between the hardware and smartphone software. Our prototype thus enables convenient $\mathrm{SpO}_{2}$ measurement over the entire body, while maintaining accuracy comparable to commercial device. Its smartphone application enables

${ }^{*}$ Corresponding author.

How to cite this paper: Ovadia-Blechman, Z., Gino, O., Dandeker, L., Sheffer, N., Baltaxe, E. and Aharonson, V. (2016) The Feasibility of Flat, Portable and Wireless Device for Non-Invasive Peripheral Oxygenation Measurement over the Entire Body. J. Biomedical Science and Engineering, 9, 147-159. http://dx.doi.org/10.4236/jbise.2016.93011 
accessible and understandable results display to patients, caregivers and healthcare professionals. The application's display and alert calibration flexibility facilitates the prototype's usage in changing medical requirements and for various disease and conditions. A device based on this prototype can monitor continuously and accurately patients' $\mathrm{SpO}_{2}$ without limiting their everyday activities or disturbing their sleep and can thus significantly improve their medical care in both clinics and home.

\section{Keywords}

Peripheral Oxygen Saturation, Noninvasive Monitoring, Medical Application, Pulse Oximetry

\section{Introduction}

The transfer of oxygen from the lungs to tissue cells is carried out primarily by hemoglobin molecules in the red blood cells. The level of arterial hemoglobin oxygenation is assessed by oxygen saturation in arterial blood $\left(\mathrm{SaO}_{2}\right)$, which is the ratio of oxygenated hemoglobin concentration $\left[\mathrm{HbO}_{2}\right]$ to total hemoglobin concentration in the blood $\left(\left[\mathrm{HbO}_{2}\right]+[\mathrm{Hb}]\right)$, usually presented in percentage value (Equation (1)). Normal $\mathrm{SaO}_{2}$ at sea level is around 97\% [1] [2].

$$
\mathrm{SaO}_{2}(\%)=\frac{\mathrm{HbO}_{2}}{\mathrm{HbO}_{2}+\mathrm{Hb}} \times 100
$$

Measuring the oxygen saturation of the blood has extensive clinical implications, for adults, children and premature babies [1]. When $\mathrm{SaO}_{2}$ ratio is measured in the cutaneous level it reflects the peripheral oxygenation level and is denoted peripheral oxygen saturation $\left(\mathrm{SpO}_{2}\right)$.

Measures of $\mathrm{SpO}_{2}$ reflect the hemodynamics of the peripheral microcirculation and thus the changes in the body oxygen supply and can indicate the disorder that triggered it (i.e. levels of hypoxia) [2].

Blood oxygen saturation assessments are routinely performed in hospitals for both screening and monitoring of patients with cardiac and pulmonary diseases, for trauma patients, in surgery and more [3]-[5]. Common diseases and conditions like chronic obstructive pulmonary disease (COPD), asthma and migraine are also associated with a disruption in the supply of oxygen to tissues in the body [6]-[8] and can benefit from oxygen saturation monitoring. Sleep disorders research and treatment can also employ continuous monitoring of blood oxidation, which can assist in the detection of sleep staging and sleep architecture. Understanding of sleep pathology is important, for example, for treatment of patients with obstructive sleep apnea (OSA) [9]-[12].

The importance of $\mathrm{SpO}_{2}$ monitoring and its wide usage in healthcare led to many efforts to devise methods that could accurately measure this index. Current device enable monitoring of the peripheral microcirculation hemodynamics variables, particularly the level of tissue oxidation [12]-[15].

Blood oxygen saturation level is commonly measured based on oximetry, known also as Near Infrared Spectroscopy (NIRS). Oximetry measures spectrophotometric changes in the optical absorption of deoxyhemoglobin $(\mathrm{Hb})$ and oxyhemoglobin $\left(\mathrm{HbO}_{2}\right)$ in the visible $(600-700 \mathrm{~nm})$ and near-infrared $(700-1000 \mathrm{~nm})$ spectral regions, respectively. The ratio of absorption between the two wavelengths determines $\mathrm{SaO}_{2}$.

The prevalent method for $\mathrm{SpO}_{2}$ measurement, close to the capillaries, is the pulse-oximeter [3] [16], which is a NIRS based measurement device. It is simple, noninvasive, and is widely used in medical institutions. The pulse oximeter is often used as part of the routine protocol of admission to emergency rooms, recovery rooms and intensive care units in hospitals, as well as in home care [16] [17].

A more elaborate NIRS-based device is the co-oximeter [18] [19] that can measure concentrations of carboxy hemoglobin and methemoglobin as a percentage of the total hemoglobin concentration in blood samples. Cooximetry is a common test for hypoxia patients as well as for patients who were exposed to low oxygen levels or high carbon levels. Its advantage is its capability to distinguish between different types of hemoglobin.

Device performing pulse oximetry and co-oximetry are non-invasive and inexpensive. However, device implementing both methods have limitations: Both use clips, which contain the light emitting device on one side and the sensor of light absorption on the other. To enable transillumination the clip has to be placed on narrow 
areas in the body, like fingertip or ear lobe (usually for newborn and premature babies) where the light can optimally cross the capillaries. The clip is uncomfortable to wear, especially for long periods. The device is also sensitive to body movement, which yield unstable signals and makes continuous monitoring inconsistent [20].

Moreover, for many of the pulse oximetry applications, home-care measurement device are advantageous: Monitoring Peripheral Oxygenation at home can allow effective diagnosis, treatment and can even be lifesaving. Implementing peripheral oxygenation measurement in home care can aid and support the routine clinical follow-up of patients, such as OSA patients receiving CPAP treatment [5]. Such device can enable untrained patients to carry out the measurement in their home and natural environment, thus maximizing patient's comfort while minimizing costs and labor [5] [21].

The limitations of conventional pulse oximeters decrease their suitability for home usage: The clip and wire of those device prohibit their usage for continuous monitoring in everyday activities at a home environment, when patients may be standing, walking, or sleeping. Most existing pulse oximetry device are uncomfortable to wear for continuous monitoring and/or are too complex to read, which equally impedes their usage for home care.

The increasing need for quality home-care device that could provide portable, non-invasive and convenient way to measure and indicate blood oxygen saturation changes has recently led to the development of several portable oxygen saturation measurement device for homecare [17] [18]. The need for wireless implementation for pulse oximetry as well as other physiological measurements for home usage led to the development of several wristwatch measurements. The growing smartphone market has also advocated an implementation of medical applications, for physiological measurements in home usage. Those applications, however, are confined to a wristwatch platform, and thus are limited for wrist measurements only [22]-[25], or based on the smartphone camera and thus are limited for finger measurements only [26].

A solution to the shape constraints of Pulse Oximeters which limit their placement locations on the body is using reflectance of light instead of absorption, which can be obtained from thicker body areas. In this method, the light emitting diode (LED) and reflectance (backscattering) light capturing device (usually a photodiode-PD) can be placed side-by-side on the same planar substrate, and thus enable readings from multiple body locations where transillumination measurements are not feasible [27]-[29].

Previous studies indicate, however, that many difficulties arise in the calibration of earlier reflectance pulse oximetry which limits their accuracy. A major challenge in reflectance oximetry is that the intensity of the backscattered light from the skin is affected not only by the optical absorption spectrum of the blood, but also by the structure and pigmentation of the skin, bones reflection, tissue backscattering and blood amount in the vascular bed. In addition, the contact between the sensor and the skin can influence the distribution of blood close to the skin surface and consequently can vary significantly between different anatomical locations [10] [16].

Another issue is the proximity of the PD to the LED in a flat reflectance based sensor configuration: If too close to each other, a PD saturation can arise. If too far, the intensity is exponentially reduced. Existing reflectance based pulse oximeters (Nellcor ${ }^{\odot}$, [30]) still use a wired configuration which decreases their usage comfort and flexibility and incorporate a small dedicated flat screen that is more suitable to healthcare professionals to read. The cost of that device is rather high for home usage and is thus limited to clinical use only.

The goal of the present study was to develop a low cost, portable, flat and wireless pulse oximeter, for home care, that could meet the following criteria:

1) Comfortable to wear in normal daily routine: A small and mobile device, which can be conveniently placed on the body and enable free movement, in daily tasks and/or sleep, without compromising adequate signal acquisition. 2) Prompt alert of an anomaly, on a small portable screen, which is easily readable and understandable to the naive user and 3) Low cost with low energy consumption.

\section{Material and Methods}

\subsection{Design and Implementation Overview}

The system implementation (Figure 1) incorporates the following elements:

1) A commercial reflectance pulse oximeter sensor (Nellcor ${ }^{\circ}$, model: MAX-FAST), characterized by its sensitivity and fast response to changes in arterial oxygen saturation during poor tissue perfusion or weak pulses. 


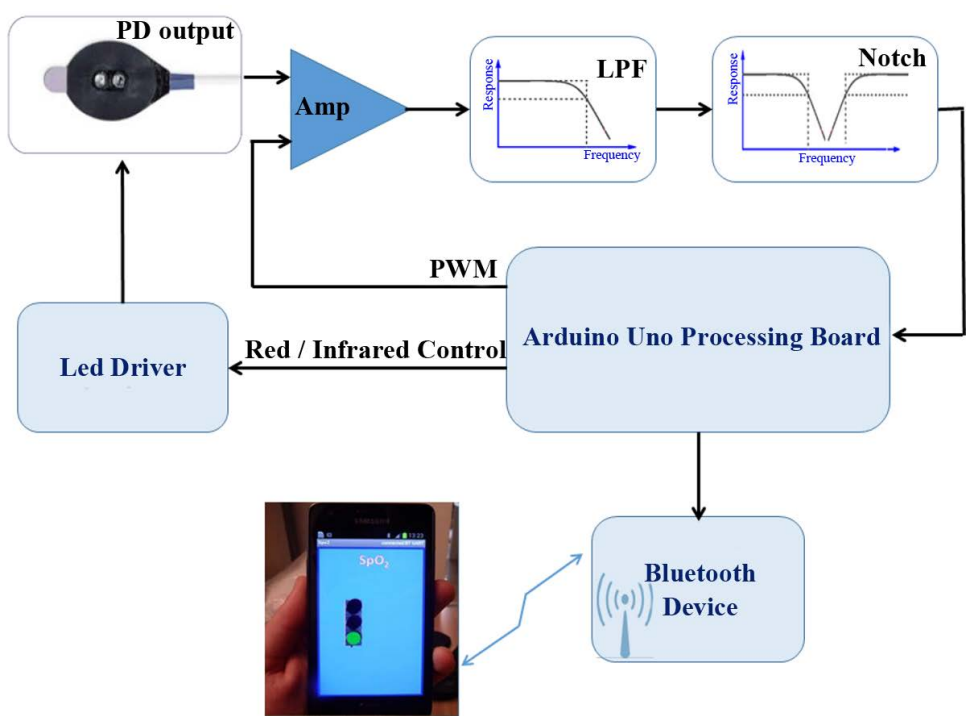

Figure 1. Prototype block diagram.

2) An Arduino Uno development kit for algorithm implementation, signal processing, synchronization and control.

3) A Front end amplifier circuit.

4) Analog filters for noise reduction.

5) A Bluetooth device.

6) A Smartphone (Android operating system).

The MAX-FAST sensor contains a pair of light emitting diodes (LEDs) that produce the required wavelengths (660 $\mathrm{nm}$ and $940 \mathrm{~nm}$ for red and infrared wavelength, respectively) and a photodiode (PD) to detect the reflected light. Since a single PD detector is used, LEDs ON/OFF switching, at a rate of $1 \mathrm{KHz}$, is implemented. The PD output signal is amplified and filtered by a Low Pass Filter followed by a Notch Filter in order to filter out noise above switching frequency of $1 \mathrm{KHz}$ and reject the network's $50 \mathrm{~Hz}$ frequency. Front end Transimpedanceamplifier (TI-OPA380) is used to convert the low-level PD currents to usable voltage signals. This amplifier is ideally suited for high-speed photodiode applications and provides very low-noise characteristics and wide dynamic range of 4 to 5 decades, which allows for measurement of currents from $1 \mathrm{nA}$ up to $100 \mu \mathrm{A}$. The Electrical circuitry description and drawing are provided in Appendix A.

The signal processing algorithms were implemented on an Arduino Uno board using Arduino Uno development kit. A Bluetooth device transmits the Arduino outputs to a smartphone. An Android's application was developed to display the outputs on the smartphone's screen, in a user friendly graphical user interface. The system's power source comprises of two $5 \mathrm{~V}$ batteries.

\subsection{Signal Acquisition and Processing}

The control of the signals acquisition, as well as the $\mathrm{SpO}_{2}$ computation from those signals was implemented on the Arduino Uno board.

Since there is only one PD in the reflectance pulse oximeter sensor, the red and IR LEDs were switched on and off intermittently to obtain alternating samples for both signals. The switching frequency was set to $1[\mathrm{KHz}]$, twice the maximal Arduino Uno's Pulse Width Modulation (PWM) frequency $(512 \mathrm{~Hz})$. This frequency is also well above the maximum arterial pulse frequency, and thus avoids the misdetection of oxygen level changes. To ensure adequate sampling of both signals, a blank time period was set between red/IR LED activation [27] [28]. The light current dispersion time is between $10-100 \mu \mathrm{sec}$, therefore this blank time was set to $250 \mu \mathrm{sec}$ (Figure 2).

The filtered signals were processed to obtain the $\mathrm{SpO}_{2}$, similarly to commercial pulse oximetry device [5] [27]:

The $\mathrm{SpO}_{2}$ was computed as a normalized ratio R (Equation (2)) of the AC component to the DC component of each light source-red and infrared (IR)-signal (Equation (2)). 




Figure 2. The prototype red (R) and infrared (IR) LED control signals.

$$
R=\frac{A C_{R E D} / D C_{R E D}}{A C_{I R} / D C_{I R}}
$$

The algorithm detects and verifies the two maxima values, corresponding to the AC and DC components, in the red and IR time domain signals. The ratio $\mathrm{R}$ is calculated for every pulse of the two light sources, yielding a $\mathrm{SpO}_{2}$ signal. For accurate reading of the $\mathrm{SpO}_{2}$ signal, weighted moving average (WMA) smoothing filters were implemented [13].

\subsection{Smartphone Application}

In order to reduce cost and enhance usability, the results of the signals processing and computation of the device are presented to the user on a smartphone screen. An Android application was developed to communicate with the device using a Bluetooth protocol and to provide a user-friendly display of the measurements’ results.

In order to present the results in a clear yet non-complex manner for non-professional users, an intuitive "traffic light” graphic user interface (GUI) was designed (Figure 3).

The "red light" in the traffic light figure indicates critical levels $\left(\% \mathrm{SpO}_{2}<90 \%\right)$, a "green light" indicates normal $\mathrm{SpO}_{2}$ level (>95\%) and a "yellow light” indicates an intermediate interval values between $90 \%$ and $94 \%$ $\mathrm{SpO}_{2}$.

The intervals classification was set according to clinical values, based on the hemoglobin dissociation curve. The red light display is accompanied by a phone vibration. The GUI also displays the numeric values of $\mathrm{SpO}_{2}$ levels. The traffic light intervals (critical, normal and intermediate), can be easily adjusted using the interface setting menu, according to medical needs and criteria.

\subsection{System Tests}

The reliability of the measurement was tested using two methods: 1) The correlation of the prototype's signals spectrum to heart rate frequencies measurements of commercial device, indicating its ability to reflect changes caused by blood pulses that originated in the heart. 2) The correlation of the $\mathrm{SpO}_{2}$ calculations of the prototype's to measurements of commercial device, in normal as well as induced anomaly in blood flow.

Since this is only a proof of concept for the design, the testing process was performed on a single subject, a healthy non-smoker female.

\subsubsection{Heart Rate Frequency Measurements}

The testing setup for the heart rate frequency correlations included heart rate monitoring of the peripheral signals produced by our $\mathrm{SpO}_{2}$ prototype, with simultaneous monitoring by a Photoplatismograph (PPG) pulse sensor. The measurement took one minute. The spectra of both signals were computed to obtain a peripheral and optical heartbeat pulse measurement (Figure 4). Those spectra were also compared to the frequencies of the electrical heart activity, which was recorded concurrently by standard electrodes’ Electrocardiography (ECG). Both ECG and PPG signals were acquired using BIOPAC ${ }^{\mathrm{TM}}$ system (BIOPAC Systems, Inc., USA). 


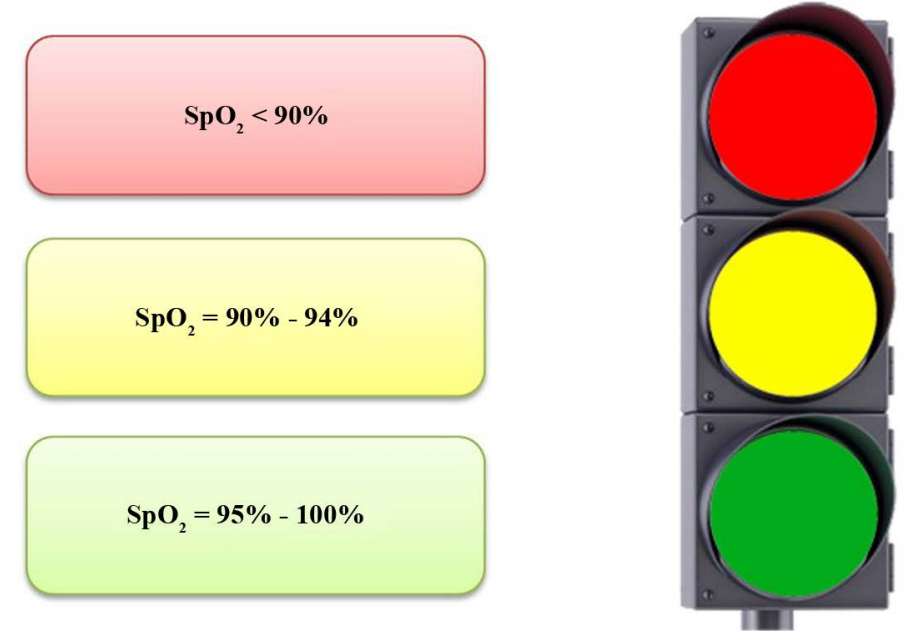

Figure 3. The "traffic light" design of the Smartphone application display and the color coding for the $3 \mathrm{SpO}_{2}$ levels.

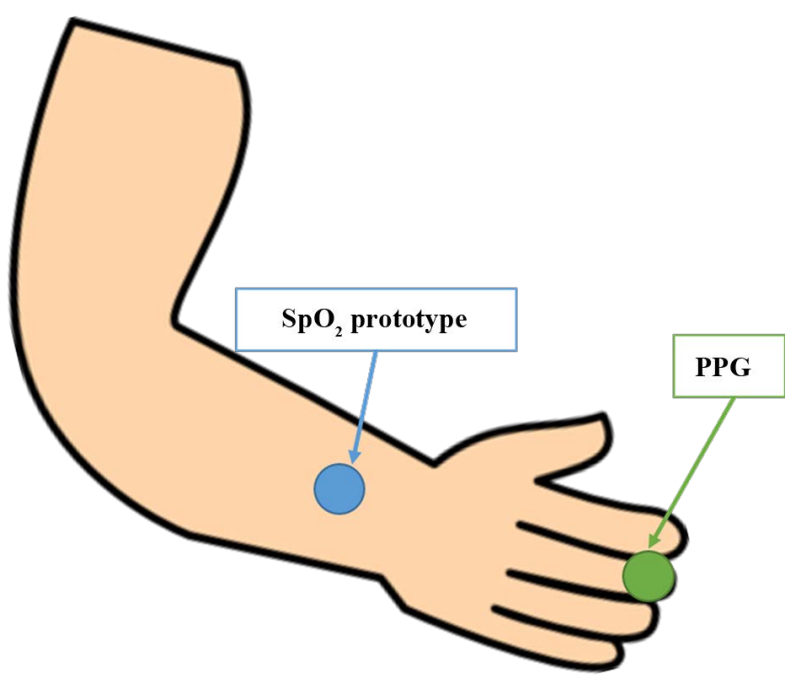

Figure 4. Testing setup for the correctness of the $\mathrm{SpO}_{2}$ prototype heart rate frequency measurement.

The BIOPAC outputs were acquired using the BIOPAC's software and the $\mathrm{SpO}_{2}$ prototype signals were sampled by an oscilloscope. The spectrum calculation for all 3 signals was performed by fast Fourier transform (FFT) using Matlab ${ }^{\odot}$.

\subsection{2. $\mathrm{SpO}_{2}$ Measurements Sensitivity}

The sensitivity of the prototype's $\mathrm{SpO}_{2}$ level computation was tested by the following setup (Figure 5). The $\mathrm{SpO}_{2}$ level was monitored by the prototype, concurrently with $\mathrm{SpO}_{2}$ measurements of a commercial pulse oximeter (Check Mate ${ }^{\mathrm{TM}}$, SPO Medical Ltd., Israel). Simultaneously, transcutaneous oxygen tension $\left(\mathrm{tcPO}_{2}\right)$ was monitored using a PeriFlux system (PERIMED ${ }^{\mathrm{TM}}$, Sweden). This latter measurement is considered as reliable and sensitive to changes in oxygen supply to the tissue and is used in clinical assessments.

In order to monitor changes in the levels of oxygen saturation, the measurements were taken during low tissue perfusion and reperfusion conditions. For this purpose, a tourniquet was placed at the subject's elbow, as shown in Figure 5, to induce tissue hypoxia. The measurements were continuously performed through a baseline period (corresponding to normal physiological state of the subject), followed by a period when the tourniquet was tightened on the subject's arm (corresponding to tissue hypoxia caused by low perfusion) and a period when the 


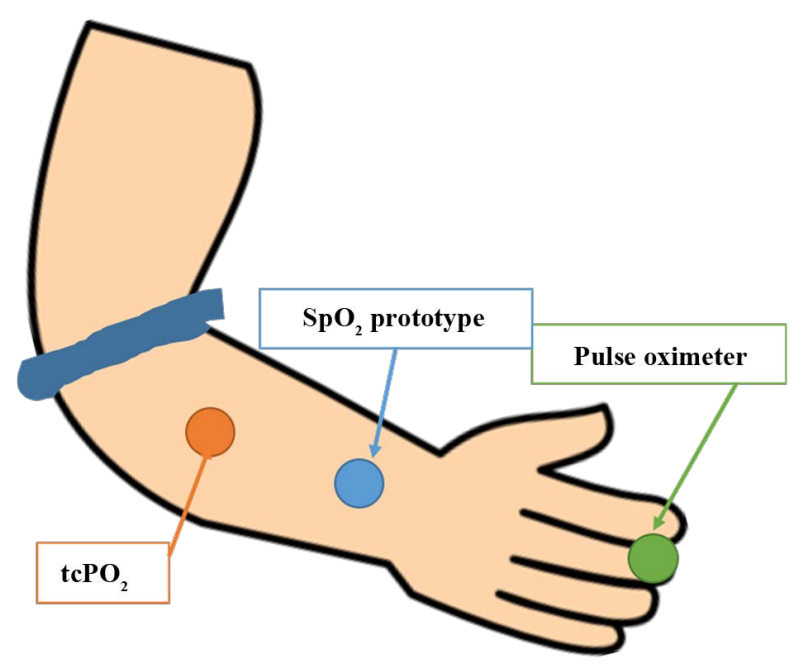

Figure 5. Testing setup for the comparison of the prototype $\mathrm{SpO}_{2}$ measurement to commercial devices measurements of $\mathrm{SpO}_{2}$ and $\mathrm{tcPO}_{2}$.

tourniquet was removed from the subject's arm (corresponding to reperfusion state). The tc $\mathrm{PO}_{2}$ and the $\mathrm{SpO}_{2}$ values were monitored and sampled every 10 seconds. The measurement took two minutes. The oxygen tension and oxygen saturation device have, however, different measurement units. In order to compare the values of the two measurements, a "Z"-normalization (Z-score), given by Equation 3, was applied for each measurement, where $\mathrm{s}$ is the non-normalized measurement value, and $\mu$ and $\sigma$ are the mean and standard-deviation of its distribution, respectively.

$$
\text { Z-score }=(s-\mu) / \sigma
$$

\section{Results}

\subsection{Heart Rate Frequency Measurements}

The signals obtained by our prototype and the two commercial sensors: ECG and PPG acquired by the BIOPAC system are demonstrated in Figure 6. The graphs at the left hand side of the figure present the signals in time domain and the graphs at the right hand side present their corresponding spectra.

It can be seen that in all three sensors the dominant frequency in the spectrum is approximately $1[\mathrm{~Hz}]$ which corresponds to a heart rate of 60 [BPM]. This heart rate fits the expected values for a relaxed healthy subject with no physical effort.

This result indicates that the prototype measurements reflect changes caused by blood pulses originated in the heart.

\section{2. $\mathrm{SpO}_{2}$ Measurements Sensitivity}

The simultaneous measurements of our prototype, the commercial $\mathrm{SpO}_{2}$ measurements and the $\mathrm{tcPO}_{2}$ measurements are demonstrated in Figure 7. The Y-axis values are normalized Z-scores of the measurements. The $\mathrm{X}$-axis indicates the simultaneous measurements timeline. Samples are presented in $10 \mathrm{sec}$ intervals, where the 0 - $30 \mathrm{sec}$ interval corresponds to a resting, baseline state. Samples 40 - 60 are values measured after tightening the tourniquet, simulating hypoxia. Samples 70 - 110 are values taken after the tourniquet was released, a reperfusion state.

The tc $\mathrm{PO}_{2}$ values clearly reflect the hypoxia and reperfusion effects. The prototype measurements show a more abrupt change at the transition from baseline $\mathrm{SpO}_{2}$ levels to simulated hypoxia $\mathrm{SpO}_{2}$ levels. It was thus successful in identifying the oxygen changes that occurred following physiological changes in oxygenation, that were observed in the tc $\mathrm{PO}_{2}$ measurements. The prototype $\mathrm{SpO}_{2}$ values as well as the overall trend of change following physiological changes in oxygenation were similar in most time intervals to the commercial device. There are still differences in the measurement values in the transition intervals between "baseline" and "hypoxia" (at 

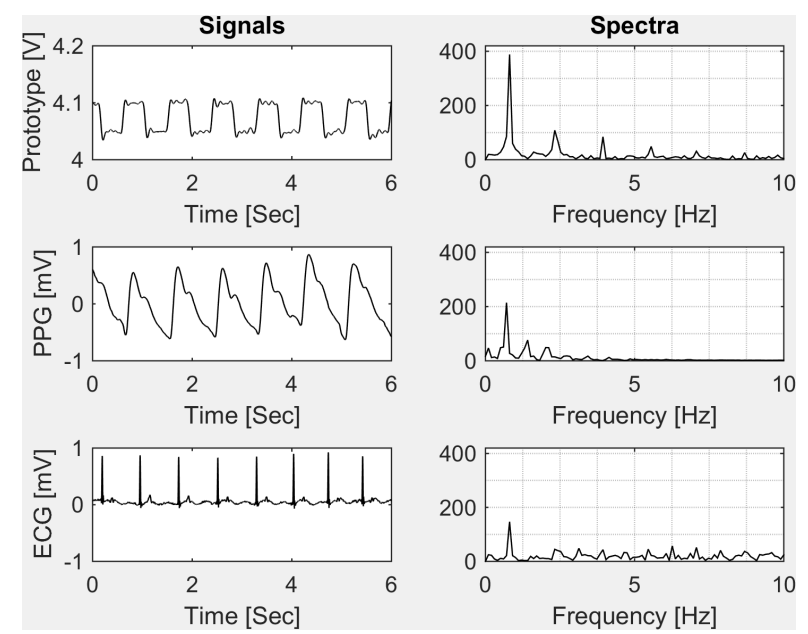

Figure 6. Heart rate frequency estimation by the 3 sensors: the new prototype (upper panels) BIOPAC PPG (middle panels) and ECG (bottom panels). Signals in time domain are plotted in the left hand side panels and their respective FFT spectra in the right hand side panels.

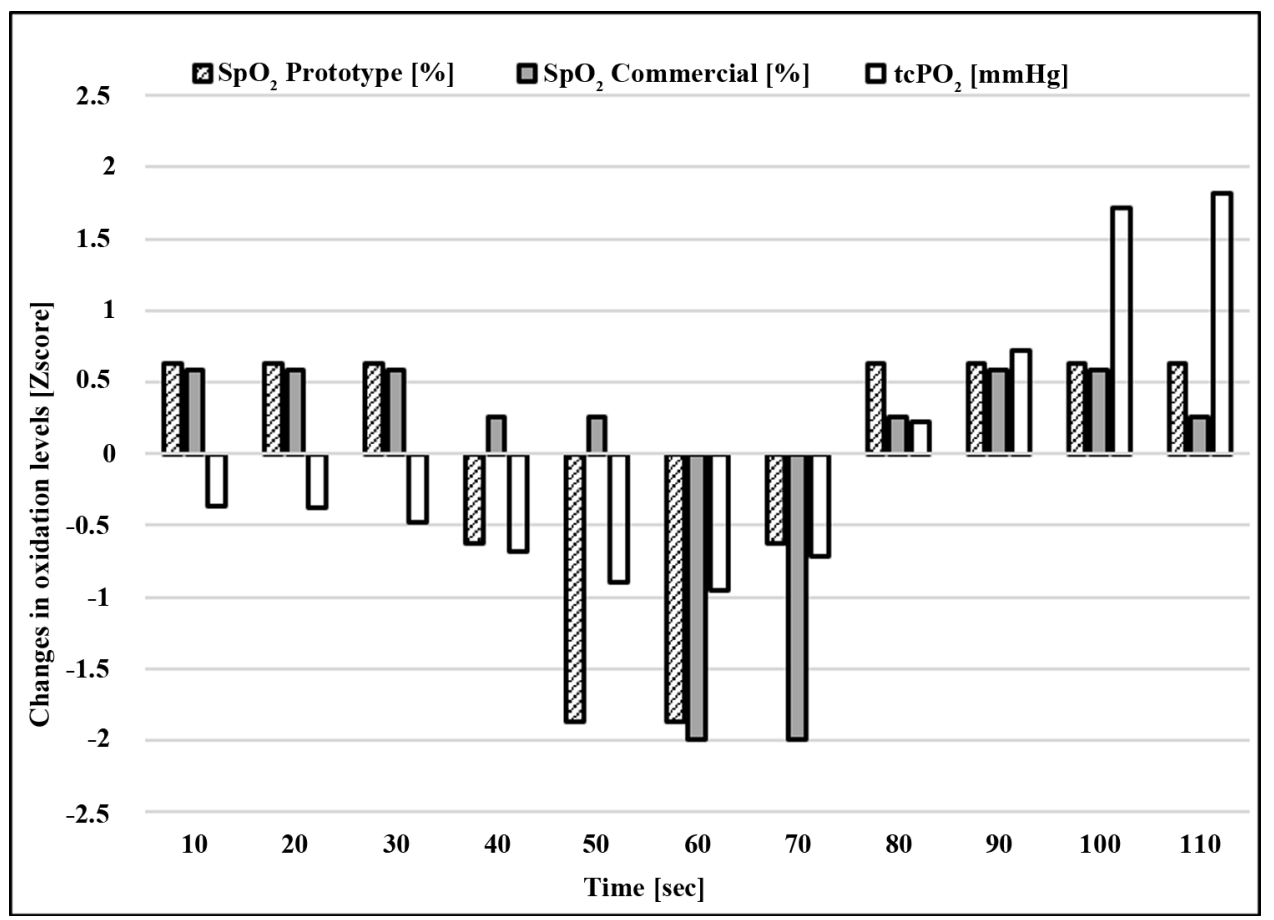

Figure 7. $\mathrm{Z}$ scores of the oxidation levels, measured by the two sensors and $\mathrm{tcPO}_{2}$ levels. The $\mathrm{X}$-axis indicates the measurement timeline, where samples 0 - $30 \mathrm{sec}$ are baseline values, samples 40 - 60 are values taken after tightening the tourniquet and samples 70 - 110 are values measured when the tourniquet was released.

40 - $50 \mathrm{sec}$ ) and between "hypoxia” and "reperfusion” (at $70 \mathrm{sec}$ ). The "steady state” values of "baseline", "hypoxia" and "reperfusion", at 0 - 30, 50 and 80 - $100 \mathrm{sec}$, respectively, are similar in both device's measurements.

\subsection{System Display Synchronization}

The electronic circuitry performance demonstrated real time synchronization to the smartphone's application. Figure 8 shows the changing of the traffic light's color on the GUI, simultaneously and wirelessly, with the appropriate light bulb in the electrical circuit. 




Figure 8. Electrical circuit and Smartphone application display.

\section{Discussion}

Pulse oximetry plays an important role in monitoring the health of patients and is widely used in hospitalization wards, intensive care units, operation rooms, birth and neonatal care, sleep laboratories, and home care [13].

The goal of this study was to develop a low cost, portable, flat and wireless device for measuring oxygenation saturation that could be usable for home care, in daily life routine as well as during sleep.

Our portable and wireless design is able to meet those challenges. The design consists of a single MAXFAST PD sensor, incorporated in an electrical circuit. The system enables instantaneous detection of both red and IR wavelengths by synchronized switching. Signal processing and control algorithms are implemented on an Arduino Uno development kit. A Bluetooth device and a smartphone application enable comfortable usage and user friendly interface. This design yields an efficient, wireless, user friendly and low cost prototype.

Our prototype is flat and based on reflected light measurements. Therefore, it enables a more comfortable yet reliable pulse oximetry on various body areas. In reflectance pulse oximetry sensors, however, both LED and PD are placed on the same plane. A trade-off of optimal distance between LEDs and PD needs to be considered: Placing the PD farther apart from the LEDs enables more separation on both red and IR relative pulse amplitude measurements (AC \& DC) but higher LED driving current is required to overcome absorption due to increase of optical path length and backscattering from epidermal layers in the skin. The commercial Nellcor ${ }^{\odot}$ reflectance sensor (model: MAX-FAST) used in our prototype incorporates this trade-off in the distance between LEDs and $\mathrm{PD}$, and its power management and signals amplification are corrected by our circuitry.

The power consumption of a pulse oximeter is dominated by the current required to drive the LEDs. The current determines the effective penetration depth of the incident light and the light intensity. Reflected signals received at the PD have generally lower amplitudes compared to transmittance mode pulse oximetry. The amplification circuit in our prototype and the signal processing performed on the signals were effective in overcoming the low amplitude, noise and artifacts in the signals.

Another key requirement for a portable pulse oximeter is efficient power management [26] [31]. In our prototype, the MAX-FAST sensor output is processed by a trans impedance amplifier that is characterized by lownoise and high dynamic range and provides adequate signal-to-noise ratio (SNR) for the required sensitivity for AC \& DC pulse amplitude detection. The design of our prototype also incorporates a power management that enables low power consumption without compromising signal quality.

Another challenge in the design was choosing the placement of the device on the body: Backscattered light intensity can vary significantly between different anatomical locations. Optical reflectance from the forehead region is typically stronger because of the relatively thin skin covering the skull combined with a higher density of blood vessels. Other anatomical locations, such as the limbs or torso, have a much lower density of blood vessels and in addition lack a dominant skeletal structure in close proximity to the skin that can help in reflecting some of the incident light. Therefore, the AC components of the reflected signal from these body locations are considerably smaller. Consequently, it is more difficult to perform accurate pulse oximetry measurement from these body locations without enhancing cutaneous circulation [16]. 
To investigate the sensitivity of the prototype, it was necessary to measure the oxygen saturation in different states of tissue perfusion. Since the physiological states of hypoxia and reperfusion can't be induced on the forehead, the sensor was placed on the forearm and a tourniquet placed at the elbow, proximally to the sensor, as shown in Figure 5.

The preliminary results indicate that the prototype device can reflect changes caused by blood pulses according to the heart rate and that it is sensitive to changes in oxygen saturation. The $\mathrm{tcPO}_{2}$ method, which measures tissue oxygen tension, is more sensitive to the vasomotion of the microcirculation system. Vasomotion indicates the changes in blood flow which is controlled by the microcirculation system itself which the $\mathrm{SpO}_{2}$ method is less sensitive to. It is important to note that $\mathrm{SpO}_{2}$ measurement device should reflect changes in the blood oxygenation and not in the blood pressure volume or flow. Due to the flat structure, the sensor could be placed near the transcutaneous oxygen tension sensor. The position of the commercial sensor was more peripheral, since it is limited only to the nail area. The distance between the two saturation sensors placement may explain the slight differences in values between them, especially the lag in the manifestation of low tissue perfusion and reperfusion conditions in the commercial device measurements.

The user interface of our prototype was designed to be very user-friendly. The measurements are displayed in an intuitive traffic light icon, in addition to displaying values, and facilitate an understanding of the data even by those who are not healthcare professionals. The light color produced in the circuit based on the instantaneous measurements is immediately transmitted and displayed on the smart phone screen, in the traffic light scheme. The mobile application also incorporates an alert when an oxygen saturation level decreases below a pre-defined level. This alert can be easily communicated by the smartphone's internet or cellular communication channels to the medical staff and could lead to faster and more efficient treatment in hospitals as well as in home care. The interface allows for flexibility in both display and alert calibration, according to the medical needs. These features can enable healthcare professionals to supply individualized care as well as to broaden the use of such device to a variety of physiological and pathological conditions. Our measurements were carried out on a single healthy subject while inducing hypoxia and reperfusion, as a preliminary testing of the prototype.

The clinical implications and possibilities will be explored in later studies, in clinical trials on healthy subjects as well as on patients with various pathologies, in hospitals as well as in home care.

Further development of the prototype should also include miniaturization of the circuitry into a small electronic chip which will further enhance the comfort, ease of placement and self-activation by the user on any part of the body.

\section{Clinical Applications}

Our prototype has the potential to substantially enhance the monitoring and treatment of chronic patients. It enables continuous monitoring of the patients' saturation without limiting their everyday activities. The system could be useful also for patients with sleep disorders such as sleep apnea, as well as for monitoring breathing of infants during sleep, and alert in case of risk to the patient due to low $\mathrm{SpO}_{2}$ level.

The flat shape of the sensor allows monitoring oxidation rates following peripheral treatment, such as skin flap and transplantation of fingers. This monitoring could diagnose and alter, as early as possible, acceptance or rejection of the graft.

Furthermore, this structure allows to monitor oxygen saturation parallel to other hemodynamics variables, such as flux and velocity of red blood cells (by Laser Doppler Flowmeter), and thus to obtain comprehensive information on the organ function.

The usability of the mobile application can be further enhanced by the various capabilities of the Smartphone: For example, based on the data derived from the measurements, an emergency call can be initialted by the device, combined with a GPS location, when the saturation level drops below a critical value. This critical value can be determined by the medical staff for each patient, enabling individualized medicine. The data and information could be securely archived in a virtual database together with the physician's inputs and patient's health records which the medical staff can conveniently access for better Tele-health treatment. This ability can provide cost-effective strategy to support the routine clinical follow-up of patients, such as OSA patients receiving CPAP treatment [21].

In line with other Tele health paradigms [26] [31], a device based on our prototype can offer a more effective diagnosis and treatment in homecare and can be even lifesaving, while reducing medical labor and costs. The system may be useful in conditions of exposure to low oxygen levels, such as during mountain climbing. In ad- 
dition, such a device could be useful in military practice.

\section{Conclusion}

This study presented a proof of concept for a portable, flat, low cost and wireless prototype for non-invasive $\mathrm{SpO}_{2}$ measurement device. The device usage is not limited to narrow areas in the body, like fingertip, ear lobe or wrist and can perform its measurements over the entire body. The proposed device is conveniently wireless, implementing Bluetooth communication and reports its results in a used-friendly, easily understood manner. This preliminary report has included a preliminary testing for the device feasibility and measurement correctness. The device could improve health care of patients with various pathologies in hospitals as well as in home care.

\section{Acknowledgements}

The authors thank engineer Eliahu Nevo, from Afeka College, for his substantial assistance in building the prototype and engineer Shalom Blechman for his contribution to the professional editing of this manuscript.

\section{References}

[1] Pittman, R.N. (2013) Oxygen Transport in the Microcirculation and Its Regulation. Microcirculation, 20, 117-137. http://dx.doi.org/10.1111/micc.12017

[2] Ovadia-Blechman, Z., Meilin, A., Rabin, N., Eldar, M. and Castel, D. (2015) Noninvasive Monitoring of Peripheral Microcirculatory Hemodynamics under Varying Degrees of Hypoxia. Respiratory Physiology \& Neurobiology, 216, 23-27. http://dx.doi.org/10.1016/j.resp.2015.05.011

[3] Kamat, V. (2002) Pulse Oximetry. Indian Journal of Anaesthesia, 46, 261-268.

[4] Mack, E. (2007) Focus on Diagnosis: Co-Oximetry. Pediatrics in Review/American Academy of Pediatrics, 28, 73. http://dx.doi.org/10.1542/pir.28-2-73

[5] Jubran, A. (1999) Pulse Oximetry. Crit Care, 3, R11-R17. http://dx.doi.org/10.1186/cc341

[6] Amery, W.K. (1985) Migraine and Cerebral Hypoxia: A Hypothesis with Pharmacotherapeutic Implications. Cephalalgia, 5, 131-133.

[7] Rodriguez-Roisin, R., Drakulovic, M., Rodríguez, D.A., Roca, J., Barbera, J.A. and Wagner, P.D. (2009) VentilationPerfusion Imbalance and Chronic Obstructive Pulmonary Disease Staging Severity. Journal of Applied Physiology, 106, 1902-1908. http://dx.doi.org/10.1152/japplphysiol.00085.2009

[8] Teague, W.G., Tustison, N.J. and Altes, T.A. (2014) Ventilation Heterogeneity in Asthma. Journal of Asthma, 51, 677684. http://dx.doi.org/10.3109/02770903.2014.914535

[9] Hedner, J., White, D.P., Malhotra, A., Herscovici, S., Pittman, S.D., Zou, D., Grote, L. and Pillar, G. (2011) Sleep Staging Based on Autonomic Signals: A Multi-Center Validation Study. Journal of Clinical Sleep Medicine: JCSM: Official Publication of the American Academy of Sleep Medicine, 7, 301.

[10] Ramirez, J.-M., Garcia, A.J., Anderson, T.M., Koschnitzky, J.E., Peng, Y.-J., Kumar, G.K. and Prabhakar, N.R. (2013) Central and Peripheral Factors Contributing to Obstructive Sleep Apneas. Respiratory Physiology \& Neurobiology, 189, 344-353. http://dx.doi.org/10.1016/j.resp.2013.06.004

[11] Levy, P., Pépin, J., Arnaud, C., Tamisier, R., Borel, J., Dematteis, M., Godin-Ribuot, D. and Ribuot, C. (2008) Intermittent Hypoxia and Sleep-Disordered Breathing: Current Concepts and Perspectives. European Respiratory Journal, 32, 1082-1095. http://dx.doi.org/10.1183/09031936.00013308

[12] Wright, C., Kroner, C. and Draijer, R. (2006) Non-Invasive Methods and Stimuli for Evaluating the Skin’s Microcirculation. Journal of Pharmacological and Toxicological Methods, 54, 1-25. http://dx.doi.org/10.1016/j.vascn.2005.09.004

[13] Liu, H., Ivanov, K., Wang, Y. and Wang, L. (2015) A Novel Method Based on Two Cameras for Accurate Estimation of Arterial Oxygen Saturation. BioMedical Engineering OnLine, 14, 52. http://dx.doi.org/10.1186/s12938-015-0045-1

[14] Nitzan, M. and Engelberg, S. (2009) Three-Wavelength Technique for the Measurement of Oxygen Saturation in Arterial Blood and in Venous Blood. Journal of Biomedical Optics, 14, 024046. http://dx.doi.org/10.1117/1.3120496

[15] De Backer, D., Donadello, K. and Cortes, D.O. (2012) Monitoring the Microcirculation. Journal of Clinical Monitoring and Computing, 26, 361-366. http://dx.doi.org/10.1007/s10877-012-9383-8

[16] Nitzan, M., Romem, A. and Koppel, R. (2014) Pulse Oximetry: Fundamentals and Technology Update. Medical Devices (Auckland, NZ), 7, 231. http://dx.doi.org/10.2147/mder.s47319

[17] Hedner, J., Pillar, G., Pittman, S.D., Zou, D., Grote, L. and White, D.P. (2004) A Novel Adaptive Wrist Actigraphy 
Algorithm for Sleep-Wake Assessment in Sleep Apnea Patients. Sleep, 27, 1560-1566.

[18] Barker, S.J., Curry, J., Redford, D. and Morgan, S. (2006) Measurement of Carboxyhemoglobin and Methemoglobin by Pulse Oximetry: A Human Volunteer Study. The Journal of the American Society of Anesthesiologists, 105, 892897.

[19] Feiner, J.R., Bickler, P.E. and Mannheimer, P.D. (2010) Accuracy of Methemoglobin Detection by Pulse CO-Oximetry during Hypoxia. Anesthesia \& Analgesia, 111, 143-148. http://dx.doi.org/10.1213/ANE.0b013e3181f46da8

[20] Krishnan, R., Natarajan, B. and Warren, S. (2010) Two-Stage Approach for Detection and Reduction of Motion Artifacts in Photoplethysmographic Data. IEEE Transactions on Biomedical Engineering, 57, 1867-1876. http://dx.doi.org/10.1109/TBME.2009.2039568

[21] Isetta, V., Negrín, M.A., Monasterio, C., Masa, J.F., Feu, N., Álvarez, A., Campos-Rodriguez, F., Ruiz, C., Abad, J. and Vázquez-Polo, F.J. (2015) A Bayesian Cost-Effectiveness Analysis of a Telemedicine-Based Strategy for the Management of Sleep Apnoea: A Multicentre Randomised Controlled Trial. Thorax, 70, 1054-1061. http://dx.doi.org/10.1136/thoraxjnl-2015-207032

[22] Patel, J. (2014) Vital Technology. XRDS: Crossroads. The ACM Magazine for Students, 21, 11. http://dx.doi.org/10.1145/2684375

[23] Rawassizadeh, R., Price, B.A. and Petre, M. (2014) Wearables: Has the Age of Smart Watches Finally Arrived? Communications of the ACM, 58, 45-47. http://dx.doi.org/10.1145/2629633

[24] Eisen, L., Fine, I. and Goldinov, L. (2012) Wearable Pulse Oximetry Device. US Patent Application No. 14/239578.

[25] Ehrler, F. and Lovis, C. (2013) Supporting Elderly Homecare with Smart Watches: Advantages and Drawbacks. Studies in Health Technology and Informatics, 205, 667-671.

[26] Scully, C.G., Lee, J., Meyer, J., Gorbach, A.M., Granquist-Fraser, D., Mendelson, Y. and Chon, K.H. (2012) Physiological Parameter Monitoring from Optical Recordings with a Mobile Phone. IEEE Transactions on Biomedical Engineering, 59, 303-306. http://dx.doi.org/10.1109/TBME.2011.2163157

[27] Mendelson, Y. and Ochs, B.D. (1988) Noninvasive Pulse Oximetry Utilizing Skin Reflectance Photoplethysmography. IEEE Transactions on Biomedical Engineering, 35, 798-805. http://dx.doi.org/10.1109/10.7286

[28] Bagha, S. and Shaw, L. (2011) A Real Time Analysis of PPG Signal for Measurement of SpO2 and Pulse Rate. International Journal of Computer Applications, 36, 45-50.

[29] Yan, Y.-S., Poon, C.C. and Zhang, Y.-T. (2005) Reduction of Motion Artifact in Pulse Oximetry by Smoothed Pseudo Wigner-Ville Distribution. Journal of Neuro Engineering and Rehabilitation, 2, 3. http://dx.doi.org/10.1186/1743-0003-2-3

[30] Plummer, J.L., Ilsley, A.H., Fronsko, R.R. and Owen, H. (1997) Identification of Movement Artefact by the Nellcor N-200 and N-3000 Pulse Oximeters. Journal of Clinical Monitoring, 13, 109-113. http://dx.doi.org/10.1023/A:1007303104811

[31] Zheng, Y.-L., Ding, X.-R., Poon, C.C.Y., Lo, B.P.L., Zhang, H., Zhou, X.-L., Yang, G.-Z., Zhao, N. and Zhang, Y.-T. (2014) Unobtrusive Sensing and Wearable Devices for Health Informatics. IEEE Transactions on Biomedical Engineering, 61, 1538-1554. http://dx.doi.org/10.1109/TBME.2014.2309951 


\section{Appendix A: Electronic Sensor Implementation}

The prototype which is Arduino Uno processor based incorporated a Nellcor Max-Fast sensor and a red and infra-red LEDs and PD. Switching frequency was $1[\mathrm{KHz}]$, a low pass filter of $1.5[\mathrm{KHz}]$ coupled with a notch filter of 50 [Hz] (filtering out the noise caused by Mains network frequency) were implemented. A Sallen-Key topology for the notch filter of a single OP amp has been implemented.

Since the Arduino Uno processor can only produce digital output, a square wave of the on/off switching signal, corresponding to a voltage values ranging between full on (5 [V]) and off (0 [V]), a Pulse Width Modulation (PWM) signal was used to generate analog results using digital processing.

\section{Bluetooth Device}

To afford portability and comfort, the final measurements results were transmitted from the device to external visualization using Bluetooth device. Bluetooth has no power consumption compared to Wi-Fi. For the communication implementation an Arduino’s Slave Bluetooth Module Wireless Board was connected. The transmission Baud rate is 9600 [bps].

The electrical diagram of the prototype implementation is shown in Figure A1.

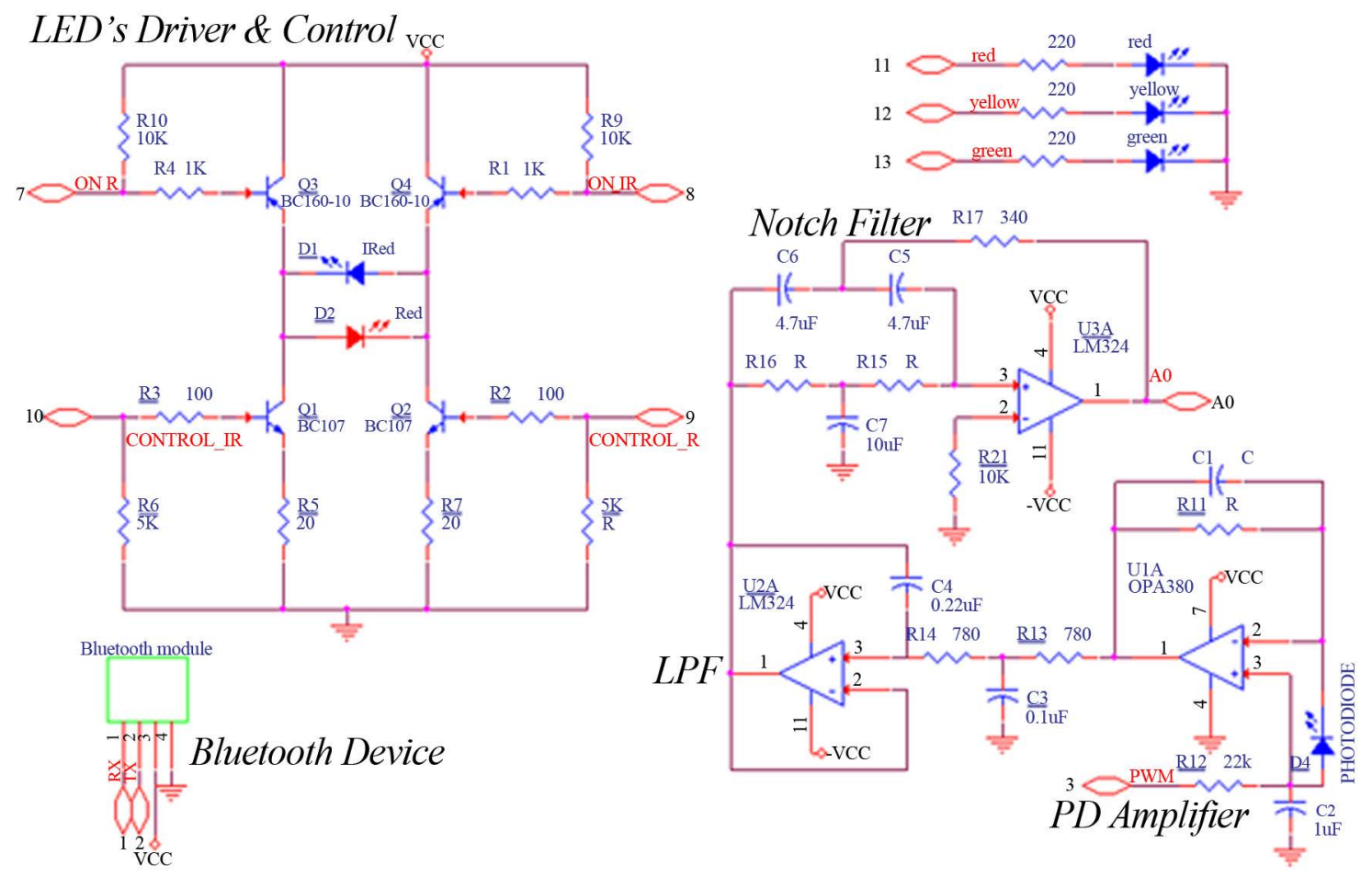

Figure A1. Electrical circuitry drawing of the sensor implementation in the prototype. 